\title{
Mineração
}

\section{Aproveitamento econômico das lamas de ferro}

\author{
(Economical recovery of iron slimes)
}

\section{Resumo}

A mineração iniciou suas atividades lavrando depósitos de alto teor, sem preocupação em maximizar seu aproveitamento. Ainda hoje, milhões de toneladas de minerais úteis são descartados anualmente para as barragens de rejeitos. Nesse trabalho, foram realizados testes de deslamagem e flotação com lamas de minério de ferro, primeiramente em escala de bancada. Várias dosagens de coletor e depressor foram utilizadas. Os resultados promissores dos testes em escala de bancada levaram a testes em escala-piloto. Foi constatado que, com o processo de flotação catiônica reversa em colunas, utilizando-se dosagem mais elevada de depressor, consegue-se recuperação mássica da ordem de $60 \%$ e $80 \%$ de recuperação metálica, 9 a $12 \%$ de Fe no rejeito, $0,55 \%$ a $0,90 \%$ de $\mathrm{SiO}_{2}$ no concentrado $\left(\mathrm{SiO}_{2}+\mathrm{Al}_{2} \mathrm{O}_{3}\right.$ $<2,00 \%$ ), além de alta seletividade no processo. A utilização da flotação de ultrafinos é uma alternativa econômica para o aumento das recuperações nos empreendimentos mineiros. A flotação de ultrafinos também pode ser avaliada para a redução ou tratamento de efluentes descartados no meio ambiente.

Palavras-chave: Flotação, ultrafinos, minério de ferro.

\section{Luciano Rocha \\ Engenheiro de Minas, M.Sc. E-mail: lucianorocha1000@hotmail.com \\ Antônio Eduardo Clark Peres \\ Professor Adjunto, Ph.D. E-mail: aecperes@demet.ufmg.br}

\section{Abstract}

High grade deposits were mined in the early stages of the activity. No attention was paid to maximizing the use of the deposits. Even nowadays, millions of tons of useful minerals are discarded every year into tailings ponds. This study investigated the possibility of economically retrieving ultrafine tailings (slimes) from an iron ore concentrator via reverse cationic flotation. The promising results of the bench scale tests led to carrying out pilot-scale tests. It was observed that a reverse cationic column flotation process, using high depressant dosage was selective, yielding high levels of iron recovery, approximately $60 \%$ mass recovery and $80 \%$ metallic recovery in the flotation stage, from 9 to $12 \%$ of $\mathrm{Fe}$ in the tailing and from $0,55 \%$ to $0,90 \%$ of $\mathrm{SiO}_{2}$ in the concentrate $\left(\mathrm{SiO}_{2}+\mathrm{Al}_{2} \mathrm{O}_{3}<2,00 \%\right)$. The ultrafines flotation is an economic alternative for increasing the recovery in mineral processing operations. The flotation of ultrafines may also be evaluated for decreasing or treating effluents disposed to the environment.

Keyword: Flotation, ultrafines, iron ore. 


\section{Introdução}

A mineração iniciou suas atividades lavrando depósitos com materiais de alto teor e sem necessidade de sofisticação no beneficiamento. Com o aumento do consumo dos bens minerais e conseqüente exaustão desses depósitos, surgiu a necessidade de técnicas mais recuperadoras para maximização das reservas minerais. Materiais de granulometria mais fina, que outrora eram descartados para o meio ambiente, hoje são rentáveis produtos. Santos (2003) destaca o caso dos rejeitos, cujo estudo tem despertado grande interesse por parte das empresas, pois, além de conduzir ao máximo de aproveitamento das jazidas, propicia um ganho ambiental, onde se busca a redução de efluentes dispostos na natureza.

Trahar (1981) estudou a relação de dependência entre o diâmetro da partícula na eficiência de coleta. Resumidamente, a eficiência de colisão é função do tamanho da partícula e a eficiência de adesão está relacionada, tanto com o grau de hidrofobicidade do mineral, quanto com o tamanho da partícula. A eficiência de colisão aumenta com o tamanho da partícula e a eficiência de adesão aumenta com o grau de hidrofobicidade e diminui com o tamanho da partícula.

Sivamohan (1990) afirma que a baixa taxa de flotação de partículas ultrafinas pode ser melhorada, aumentado a taxa de colisão e o momento de inércia. Aumentando a agregação, mantendo as bolhas menores, aumentando o tempo de residência na célula e permitindo que partículas e bolhas estejam em fluxos de sentidos contrários, aumenta-se a taxa de flotação.

Tradicionalmente, partículas muito finas não flotam (Pease et al., 2005), entretanto alguns minérios precisam de ser cominuídos a uma granulometria abaixo de $10 \mu \mathrm{m}$ para atingirem o grau de liberação necessário para a obtenção de concentrado dentro das especificações. Exemplos práticos da flotabilidade de materiais ultrafinos são a Mina MacArthur
River, onde é essencial a moagem atingindo $80 \%$ passante em $7 \mu \mathrm{m}(\mathrm{P} 80=7 \mu \mathrm{m})$, e Mt Isa, onde o P80 a ser atingido na moagem deve ficar entre $12 \mu \mathrm{m}$ e $7 \mu \mathrm{m}$. Nessas plantas, é fundamental produzir "lamas" para se atingir uma boa recuperação na flotação. Essas duas minas em conjunto produzem cerca de $1 \mathrm{Mt}$ por ano de concentrado de flotação com partículas, predominantemente, menores que $10 \mu \mathrm{m}$, obtendo acima de $80 \%$ de recuperação. A verdade é que, nessas minas, a melhor recuperação na flotação está no material ultrafino.

Uma razão para que partículas muito finas não exibam boa flotabilidade é que elas estão misturadas com partículas mais grossas. As partículas mais finas precisam de maior dosagem de coletor e maior tempo de residência, porém as condições do sistema são, geralmente, ajustadas para as partículas mais grossas.

Estima-se que $1 / 3$ da produção mundial de fosfatos, $1 / 6$ da de cobre, $1 / 5$ da de tungstênio, $1 / 2$ da produção boliviana de estanho, 1/10 da produção americana de ferro, junto com milhões de toneladas de outros minerais são perdidas nas frações ultrafinas $(<38 \mu \mathrm{m})$ (Subrahmanyam \& Forssberg, 1990; Rosa, 1997).

Esse trabalho envolve estudos da concentrabilidade de lamas de minério de ferro, através da concentração por flotação catiônica reversa e visa a obter um concentrado ultrafino a ser incorporado aos pellet feed fines tradicionais. Apesar de a lama em questão conter teor relativamente alto de contaminantes deletérios, principalmente alumina e sílica, apresenta-se liberada e com teor de ferro da ordem de $50 \%$ nas frações abaixo de $18 \mu \mathrm{m}$. A incorporação desse material ultrafino, nos concentrados de flotação, possibilitará incremento da área superficial específica dos pellet feed fines, ocasionando redução no consumo energético na moagem preparatória para a pelotização, além de aumentar o rendimento mássico do complexo. Na área ambiental reduz a geração de efluentes, prolongando a vida útil das barragens de rejeito.

\section{Materiais e métodos}

A metodologia para a realização desse trabalho baseia-se em amostragem, caracterização tecnológica e testes em escala de bancada e piloto de deslamagem e flotação.

\subsection{Amostragem}

Fez-se uma amostragem do ROM atual e foram realizados testes em escala laboratorial e piloto. Foram feitas, também, amostragens de forma a compor o ROM futuro. Considerando-se que o ROM futuro será composto por litologias que, atualmente, são descartadas como estéril, a amostragem foi feita em pilhas especialmente montadas para atender as características da lama que a empresa irá processar e também da lama que hoje é descartada para o rejeito. Com o ROM futuro foram feitos testes em escala-piloto, tendo como base os melhores resultados dos testes com o ROM atual.

\subsection{Caracterização tecnológica das lamas}

A análise da densidade real foi feita em triplicata pelo método do picnômetro, a análise química foi feita por fluorescência de raios X. A granulometria foi feita utilizando Cyclosizer, onde o tempo de lavagem foi de 5 minutos e de o elutriação, de 15 minutos, e também foi feita análise no granulômetro de difração a laser da marca Sympatec Heloscom com comprimento focal de $20 \mathrm{~mm}$. Neste, o dispersante utilizado foi o hexametafosfato de sódio, sob solução a 0,05 $\%$. Utilizou-se um permeabilímetro da marca Pavitest para determinação de área superficial específica pelo método Blaine. Utilizaram-se três técnicas BET de adsorção de gás nitrogênio: adsorção multi point, adsorção em um único ponto e Método de Boer, cujas condições de gaseificação foram de 2 horas a $200^{\circ} \mathrm{C}$ no vácuo. Para carcterização no $\mathrm{MEV}$, a amostra foi preparada em dispersão com $10 \mathrm{~mL}$ de água deionizada e $10 \mathrm{~mL}$ de dispersante (hexametafosfato de sódio) e foi agitada com bastão magnético. Foi 
adicionada uma gota no porta-amostra, com secamento natural por um dia. A metalização foi realizada com ouro (recobrimento para tornar a amostra condutora). As imagens foram obtidas através de elétrons secundários e a energia para gerálas foi de $15 \mathrm{kV}$.

\subsection{Testes em escala de bancada}

Essa fase foi planejada e realizada com o objetivo de se verificar o comportamento das lamas atualmente geradas na planta industrial. A amostra foi deslamada em duas etapas. Na primeira etapa, ele foi acondicionada em um balde de $17 \mathrm{~L}$, sob agitação manual. $\mathrm{O}$ pH da polpa foi ajustado com $\mathrm{NaOH}$ em 10,5, o material foi deixado em repouso para decantação por 5 minutos, o sobrenadante foi sifonado e coletado em um outro recipiente. Adicionou-se água no material residual do balde para ajuste da porcentagem de sólidos em $10 \%$ e repetido todo procedimento para a segunda etapa de deslamagem. O segundo sobrenadante foi incorporado ao primeiro. O Underflow alimentou a flotação em célula mecânica, o amido foi condicionado por 5 minutos a $60 \%$ de sólidos e, durante esse tempo, ajustou o $\mathrm{pH}$ a 10,70, diluiuse a polpa a $40 \%$ e adicionou-se a amina, que foi condicionada a 1 minuto. $\mathrm{O}$ tempo de flotação foi de 6 minutos.

Foram testadas algumas condições de reagentes, sendo as mais relevantes:

Amido: $600 \mathrm{~g} / \mathrm{t}$, amina: $55 \mathrm{~g} / \mathrm{t}$.

Amido: $1.000 \mathrm{~g} / \mathrm{t}$, amina: $25 \mathrm{~g} / \mathrm{t}$.

Amido: $1.000 \mathrm{~g} / \mathrm{t}$, amina: $55 \mathrm{~g} / \mathrm{t}$.

Amido: $1.200 \mathrm{~g} / \mathrm{t}$, amina: $30 \mathrm{~g} / \mathrm{t}$.

A partir do melhor resultado, foram feitos novos testes, a fim de adequar a dosagem dos reagentes.

\subsection{Testes em escala piloto}

A fase de testes-piloto contemplou duas etapas. A primeira foi realizada com as lamas provenientes do ROM atual com o objetivo de confirmação dos testes em escala de bancada. Uma segunda etapa foi realizada com as lamas provenientes do ROM futuro. A amostra foi transferida para o conjunto de ciclonagem, composto por um tanque com agitação mecânica, uma bomba e um hidrociclone de 38,1 mm (1,5 "). O hidrociclone era composto por um ápex com diâmetro de $3 \mathrm{~mm}$ e por um vortex finder com diâmetro de $10 \mathrm{~mm}$; a pressão de bombeamento foi de $147 \mathrm{kPa}$. Com base nos testes em escala de bancada, optou-se por utilizar a dosagem de $1.000 \mathrm{~g} / \mathrm{t}$ de amido e $30 \mathrm{~g} / \mathrm{t}$ de amina. $\mathrm{O}$ underflow do hidrociclone foi enviado para um tanque intermediário, onde foi ajustada a porcentagem de sólidos em $60 \%$ em massa e adicionada a solução de amido. Após o condicionamento do amido por 8 minutos, a polpa foi enviada para a caixa de bomba que alimenta a coluna de flotação. A porcentagem de sólidos foi ajustada para $40 \%$, o $\mathrm{pH}$ para 10,7 e amina foi adicionada por 1 minuto. A polpa condicionada alimentou a coluna-piloto $(4,75 \mathrm{~m}$ de altura e $100 \mathrm{~mm}$ de diâmetro) para o teste de flotação. A taxa média de alimentação, na coluna, foi de $40 \mathrm{~kg} / \mathrm{h}$ de sólidos. Visando à obtenção de uma melhor seletividade no processo, foi utilizada água de lavagem no topo da coluna, numa vazão de $80 \mathrm{~L} / \mathrm{h}$.

\section{Resultados e discussões}

\subsection{Caracterização tecnológica das lamas}

A massa específica do material foi determinada em $3,50 \mathrm{~g} / \mathrm{cm}^{3}$ com desviopadrão de 0,021 . A análise granulométrica mostrou que $85,97 \%$ da amostra era passante na malha de $7,1 \mu \mathrm{m}$ (Tabela 1 ), no cyclosizer o d90 foi igual a $10,9 \mu \mathrm{m}$ e, no granulômetro de difração a laser, o d90 foi igual a $12,2 \mu \mathrm{m}$. Os resultados obtidos pelos dois métodos (Cyclosizer e granulômetro de difração de laser) divergem por serem métodos que utilizam processos de análise completamente diferentes. O Blaine apresentou como resultado $2,768 \mathrm{~cm}^{2} / \mathrm{g}$. Os resultados obtidos de área superficial específica são os seguintes: método BET de adsorção multi point: $34,4866 \mathrm{~m}^{2} / \mathrm{g}=344.866,0$ $\mathrm{cm}^{2} / \mathrm{g}$; por adsorção em ponto único: $33,863213 \mathrm{~m}^{2} / \mathrm{g}=338.632 \mathrm{~cm}^{2} / \mathrm{g}$; método de Boer: $34,4867 \mathrm{~m}^{2} / \mathrm{g}=344.867 \mathrm{~cm}^{2} / \mathrm{g}$. Pelas três técnicas utilizadas nos ensaios BET, pode-se constatar uma boa consistência nos resultados, indicando um valor de área superficial específica em torno dos $34 \mathrm{~m}^{2} / \mathrm{g}$. É importante ressaltar a grande diferença encontrada nos resultados entre os métodos de permeabilímetro de Blaine e BET. O permeabilímetro de Blaine é um instrumento importante e muito utilizado para fins comparativos. A técnica BET tende a fornecer um valor mais alto para a área superficial específica em comparação com a técnica Blaine, pois o gás nitrogênio penetra e se adsorve nos poros e microporos das partículas e não simplesmente em sua superfície. O MEV mostrou que as partículas estavam totalmente liberadas.

\subsection{Resultados dos testes em escala de bancada}

Uma dosagem baixa de amido (600 $\mathrm{g} / \mathrm{t}$ ) não se mostrou eficiente para deprimir, satisfatoriamente, o ferro contido na polpa. Houve grande perda de ferro para o rejeito (50 a $52 \%$ ). A seletividade dos testes também se mostrou muito baixa $(1,2)$.

Os resultados com a dosagem de $1.000 \mathrm{~g} / \mathrm{t}$ de depressor e $55 \mathrm{~g} / \mathrm{t}$ de coletor foram bastante satisfatórios com relação ao teor de sílica no concentrado $(<1 \%)$, recuperações mássica e metalúrgica, além de boa seletividade no processo $(16,3)$. A fim de reduzir gastos com reagentes, a dosagem de coletor diminuiu para $25 \mathrm{~g} / \mathrm{t}$ e conseguiu-se concentrar o ferro com razoável qualidade de concentrado $(66,0 \%$ a $66,6 \%)$, porém não se obteve um teor de sílica abaixo de $1 \%$, como obtido nos testes anteriores. Houve seletividade de 10,9 na separação, menor que nos testes com dosagem de $55 \mathrm{~g} / \mathrm{t}$ de amina. Contudo resolveu-se aumentar a dosagem do depressor de $1000 \mathrm{~g} / \mathrm{t}$ para $1200 \mathrm{~g} / \mathrm{t}$ e reduzir a do coletor de $55 \mathrm{~g} / \mathrm{t}$ para $30 \mathrm{~g} / \mathrm{t}$. 
Aproveitamento econômico das lamas de ferro

Os testes com $1200 \mathrm{~g} / \mathrm{t}$ de amido e $30 \mathrm{~g} / \mathrm{t}$ de amina produziram um material que atende à especificação de pellet feed fines para produção de pelotas de redução $\operatorname{direta}\left(\mathrm{SiO}_{2}+\mathrm{Al}_{2} \mathrm{O}_{3}<2,00 \%\right.$, Tabela 2$)$.

Os testes indicaram que maiores dosagens de depressor e coletor favorecem os parâmetros avaliados, ou seja, a qualidade química no concentrado e rejeito, a recuperações mássica e metalúrgi- ca e o índice de seletividade. Os resultados da fase de bancada foram positivos o suficiente para sustentar a fase-piloto.

\subsection{Resultados dos testes em escala-piloto}

Os resultados dos testes-piloto de deslamagem e flotação com as lamas provenientes do ROM atual mostraram que é possível a obtenção de concentrados que atendam à especificação de pellet feed fines para produção de pelotas de redução direta, com recuperações acima de $60 \%$ na flotação. Em função de o material ser ultrafino, a tendência é ocorrer a flotação por arraste, mas, com o uso de água de lavagem, o processo se mostrou estável, com ótima seletividade $(28,5)$. Com a estabilidade dos testes-pi-

Tabela 1 - Análise granuloquímica.

\begin{tabular}{c|c|c|c|c|c|c|c|c}
\hline & \multicolumn{7}{|c|}{ Análise Granulométrica } & \multicolumn{7}{c}{ Análise Química (\%) } \\
\hline Malha ( $\boldsymbol{\mu m})$ & Retida (\%) & Acumulada (\%) & $\mathbf{F e}$ & $\mathbf{S i O}_{\mathbf{2}}$ & $\mathbf{A l}_{\mathbf{2}} \mathbf{O}_{\mathbf{3}}$ & $\mathbf{M n}$ & $\mathbf{P}$ & PPC \\
\hline 32,0 & 0,00 & 0,00 & - & - & - & - & - & - \\
\hline 24,5 & 0,62 & 0,62 & 25,63 & 59,89 & 0,78 & 0,01 & 0,333 & 2,40 \\
\hline 20,5 & 1,32 & 1,94 & 10,10 & 84,00 & 0,66 & 0,01 & 0,015 & 0,85 \\
\hline 12,2 & 5,64 & 7,58 & 15,93 & 75,49 & 0,70 & 0,01 & 0,017 & 1,02 \\
\hline 7,1 & 6,45 & 14,03 & 20,98 & 67,88 & 0,77 & 0,05 & 0,021 & 1,11 \\
\hline$-7,1$ & 85,97 & 100,00 & 42,88 & 28,99 & 5,11 & 0,14 & 0,081 & 4,07 \\
\hline & & Calculada & 39,41 & 35,04 & 4,50 & 0,12 & 0,072 & 3,65 \\
\hline & & Analisada & 38,23 & 37,05 & 4,36 & 0,12 & 0,071 & 3,46 \\
\hline
\end{tabular}

Tabela 2 - Flotação em Bancada, $1200 \mathrm{~g} / \mathrm{t}$ de amido e $30 \mathrm{~g} / \mathrm{t}$ de amina.

\begin{tabular}{|c|c|c|c|c|c|c|c|c|c|}
\hline \multicolumn{8}{|c|}{ Deslamagem - Amostra 05} & & \\
\hline \multirow{2}{*}{ Produtos } & \multirow{2}{*}{$\begin{array}{c}\text { Massa } \\
(\mathrm{g})\end{array}$} & \multirow{2}{*}{$\begin{array}{l}\text { Recuperação } \\
\text { mássica (\%) }\end{array}$} & \multicolumn{5}{|c|}{ Composição Química (\%) } & & \\
\hline & & & $\mathrm{Fe}$ & $\mathrm{SiO}_{2}$ & $\mathrm{Al}_{2} \mathrm{O}_{3}$ & Mn & $\mathbf{P}$ & \multicolumn{2}{|c|}{ Recuperação na Flotação (\%) } \\
\hline Alimentação & 3897,00 & 100,00 & 47,23 & 15,81 & 5,35 & 2,33 & 0,146 & Mássica & Metálica \\
\hline Underflow & 1132,00 & 29,05 & 52,78 & 21,00 & 0,81 & 0,22 & 0,026 & 72,2 & 88,1 \\
\hline Overflow & 2765,00 & 70,95 & 44,96 & 13,68 & 7,21 & 3,19 & 0,195 & \multicolumn{2}{|c|}{ Seletividade "Gaudin" } \\
\hline \multicolumn{8}{|c|}{ Flotação - Amostra 05 - 1200g/t amido e $30 \mathrm{~g} / \mathrm{t}$ amina } & & \\
\hline \multirow{2}{*}{ Produtos } & \multirow{2}{*}{$\begin{array}{c}\text { Massa } \\
(\mathrm{g})\end{array}$} & \multirow{2}{*}{$\begin{array}{c}\text { Recuperação } \\
\text { mássica (\%) }\end{array}$} & \multicolumn{5}{|c|}{ Composição Química (\%) } & \multicolumn{2}{|c|}{ Recuperação Global (\%) } \\
\hline & & & $\mathbf{F e}$ & $\mathrm{SiO}_{2}$ & $\mathrm{Al}_{2} \mathrm{O}_{3}$ & Mn & $\mathbf{P}$ & Mássica & Metálica \\
\hline Alimentação & 557,00 & 100,00 & 55,65 & 18,26 & 0,76 & 0,25 & 0,029 & 21,0 & 30,1 \\
\hline Concentrado & 402,10 & 72,19 & 67,88 & 0,76 & 0,50 & 0,31 & 0,035 & & \\
\hline Rejeito & 154,90 & 27,81 & 23,90 & 63,68 & 1,42 & 0,11 & 0,014 & & \\
\hline
\end{tabular}


Luciano Rocha et al.

Tabela 3 - Flotação em Coluna - Pilha especial com ROM Futuro.

\begin{tabular}{|c|c|c|c|c|c|c|c|c|c|}
\hline \multicolumn{8}{|c|}{ Deslamagem - Amostra 02 - Pilha Especial A } & & \\
\hline \multirow{2}{*}{ Fluxos } & \multirow{2}{*}{$\begin{array}{l}\text { Recuperação } \\
\text { mássica (\%) }\end{array}$} & \multicolumn{6}{|c|}{ Análise Química (\%) } & & \\
\hline & & $\mathrm{Fe}$ & $\mathrm{SiO}_{2}$ & $\mathrm{Al}_{2} \mathrm{O}_{3}$ & Mn & $\mathbf{P}$ & PPC & \multicolumn{2}{|c|}{ Recuperação na Flotação (\%) } \\
\hline Alimentação & 100,00 & 36,04 & 38,70 & 5,12 & 0,22 & 0,062 & 4,21 & Mássica & Metálica \\
\hline Underflow & 42,90 & 34,87 & 47,70 & 0,80 & 0,16 & 0,023 & 1,40 & 41,7 & 80,5 \\
\hline Overflow & 57,10 & 36,92 & 31,94 & 8,37 & 0,27 & 0,091 & 6,32 & \multicolumn{2}{|c|}{ Seletividade "Gaudin" } \\
\hline \multicolumn{8}{|c|}{ Flotação - Amostra 02 - Pilha Especial A } & & \\
\hline \multirow{2}{*}{ Produtos } & \multirow{2}{*}{$\begin{array}{c}\text { Recuperação } \\
\text { mássica (\%) }\end{array}$} & \multicolumn{6}{|c|}{ Composição Química (\%) } & \multicolumn{2}{|c|}{ Recuperação Global (\%) } \\
\hline & & $\mathbf{F e}$ & $\mathrm{SiO}_{2}$ & $\mathrm{Al}_{2} \mathrm{O}_{3}$ & $M n$ & $\mathbf{P}$ & PPC & Mássica & Metálica \\
\hline Alimentação & 100,00 & 34,87 & 47,70 & 0,80 & 0,16 & 0,023 & 1,40 & 17,9 & 33,4 \\
\hline Concentrado & 41,65 & 67,39 & 0,82 & 0,68 & 0,12 & 0,045 & 1,85 & & \\
\hline Rejeito & 58,35 & 11,66 & 81,16 & 0,89 & 0,19 & 0,007 & 1,08 & & \\
\hline
\end{tabular}

loto, conseguiu-se teor de ferro no rejeito bastante baixo, variando de 9 a $12 \%$.

O resultado com o ROM atual foi base para os testes com o ROM futuro. Apesar de a lama proveniente do ROM futuro conter um teor de ferro mais baixo $\mathrm{e}$, conseqüentemente, maiores teores de materiais deletérios, os testes comprovaram a alta concentrabilidade do material (Tabela 3). As recuperações foram menores que as obtidas com o ROM atual, como era de se esperar, mas ainda oferecem um valor bastante atrativo, do ponto de vista econômico. A seletividade nos testes foi alta, sendo atingidos baixos teores de Fe nos rejeitos.

\section{Conclusões}

O objetivo de se retirar um concentrado rico de minério de ferro das lamas finais da mineradora através de concentração por flotação catiônica reversa foi plenamente atingido. Os resultados dos testes mostraram que, nesse caso específico, não houve necessidade de altas dosagens de coletor. A necessidade de alta dosagem de reagente se restringiu ao depressor. Com dosagens de $1.000 \mathrm{~g} / \mathrm{t}$ de amido e $30 \mathrm{~g} / \mathrm{t}$ de amina, conseguiu-se um concentrado ultrafino com teores abaixo de $1 \%$ de sílica e teores de sílica + alumina abaixo de $2 \%$, o que qualifica os pellet feed fines para produção de pelotas de redução direta. A boa seletividade do processo possibilitou atingir teores de rejeitos da ordem de $12 \%$ de Fe, apenas no estágio rougher. Uso de colunas com água de lavagem se mostrou a condição ideal para se concentrarem ultrafinos de minério de ferro, condizendo com o exposto na literatura.

\section{Referências bibliográficas}

PEASE, J. D., CURRY, D. C., YOUNG, M. F. Designing flotation circuits for high fines recovery. Minerals Engineering, v. 19, p. 831-840, 2005.

ROSA, J. J. O condicionamento em alta intensidade e a recuperação de finos de minérios por flotação. Rio Grande do Sul: Programa de Pós-Graduação em Engenharia de Minas, Metalúrgica e de Materiais, Escola de Engenharia da Universidade Federal do Rio Grande do Sul (UFRGS), 1997. (Dissertação de Mestrado).

SANTOS, I. J. Estudo da produção de concentrados de minério de ferro a partir dos rejeitos da barragem de Germano. Ouro Preto: Escola de Minas, Universidade Federal de Ouro Preto (UFOP), 2003. (Dissertação de Mestrado).

SIVAMOHAN, R. The problem of recovering very fine particles in mineral processing - a review. International Journal of Mineral Processing. Amsterdam, Holland: Elsevier Science Publishers B. V., v. 28, p. 247-288, 1990.

SUBRAHMANYAN, T. V., FOSSBERG, E. F. S. Fine particle processing: shear flocculation and carrier flotation - a review. International Journal of Mineral Processing, v. 30, p. 265-286, 1990.

TRAHAR, W. J. A rational interpretation of role of particle size in flotation. International Journal of Mineral Processing, v. 2, p. 289-327, 1981.

Artigo recebido em 16/06/2008 e aprovado em 25/05/2009. 